\title{
Microbial redox interplay enhances biodiversity and ecosystem productivity
}

Mayumi Seto ( $\square$ seto@ics.nara-wu.ac.jp )

Nara Women's University https://orcid.org/0000-0003-4709-5206

Michio Kondoh

Tohoku University

Biological Sciences - Article

Keywords: redox reactions, chemotrophic interactions, microbial communities

Posted Date: October 19th, 2021

DOl: https://doi.org/10.21203/rs.3.rs-959776/v1

License: (c) (i) This work is licensed under a Creative Commons Attribution 4.0 International License. Read Full License 
1 Microbial redox interplay enhances biodiversity and ecosystem productivity

$2 \quad{ }^{a *}$ Mayumi Seto and ${ }^{b *}$ Michio Kondoh

3 Affiliations

$4{ }^{a}$ Department of Chemistry, Biology, and Environmental Sciences, Nara Women's

5 University, Kita-Uoya Nishimachi, Nara 630-8506, Japan

$6 \quad$ seto@ics.nara-wu.ac.jp

$7 \quad{ }^{\mathrm{b}}$ Graduate School of Life Sciences, Tohoku University, 6-3 Aramaki Aza Aoba, Aoba-ku,

8 Sendai 980-8578, Japan

Summary paragraph

A significant portion of the Earth's biodiversity and biomass is from the

12 subsurface biosphere, where chemotrophic microbial species harness diverse

13 inorganic oxidation-reduction reactions (redox reactions) as a major source of

14 metabolic energy while driving biogeochemical cycles. Given the limited

15 availability of energy in the anaerobic environment, a fundamental question concerns what interplay between the chemical environment and chemotrophic community enables the persistence of whole biogeochemical systems. Here, using a thermodynamics-based mechanistic model that maps the interplay between diverse

19 chemotrophic species and chemical compounds onto a redox network, we show

20 that cycles of redox reactions mediate chemotrophic interactions in a way that

21 increases the complexity of both redox reaction networks and microbial communities and enhances the community-level efficiency of energy metabolism.

23 The high efficiency and complexity of biogeochemical systems arises from the self-

24 organised ecological niche segmentation of microbes. More specifically, a 
consortium of chemotrophic species that subdivide a long-reaction pathway into

26 shorter-reaction segments enhance each other's population growth, replaces the

27 species that monopolises the long-reaction pathway, and increases ecosystem

28 productivity. An ecologically driven 'division of metabolic labour' in the

29 chemotrophic community provides a novel mechanism through which an intimate

30 life-environment interplay concurrently enhances biodiversity and ecosystem

31 productivity. 
Microbial cells exist up to $2.5 \mathrm{~km}$ under the surface of marine sediments ${ }^{1,2}$,

34 and the subsurface biomass contributes to a significant portion of the Earth's biomass ${ }^{3,4}$.

35 According to the previous estimations, the microbial biomass hidden beneath the

36 surface is several to tens of $\mathrm{Pg} \mathrm{C} \mathrm{C}^{3,5,6}$, which accounts for $70 \%$ of the total microbial cells

37 on Earth ${ }^{7}$. The major energy sources for the subsurface biosphere, given the limited

38 supply of light and photosynthetic products, are redox gradients (e.g., oxic-anoxic

39 interfaces or chemical gradients within deep-sea hydrothermal vent systems), which

40 provide chemical energy via redox reactions ${ }^{8-10}$.

41 The maximum energy production per redox reaction, or the negative Gibbs

42 energy change of reaction $\left(-\Delta_{r} G\right.$,), available in the subsurface anaerobic environment

43 is, in general, significantly lower than aerobic respiration. Thus, there is a question of

44 how chemotrophic microbial communities efficiently yield energy from the

45 thermodynamically less favourable environment and maintain their biodiversity ${ }^{11,12}$.

46 Current theory suggests that redox reactions can mediate mutualistic interactions

47 between chemotrophic organisms and allow for the efficient utilisation of redox

48 reactions ${ }^{13,14}$. However, earlier studies investigated relatively simple systems, thus

49 leaving the role of eco-geochemical interactions in a more realistic multi-species, multi-

50 compound system unanswered.

52 Thermodynamic understanding of chemotrophic community dynamics

To understand the eco-geochemical interplay mapped onto a complex redox

54 network, we constructed a mechanistic model that linked the population dynamics of

55 chemotrophic species to a chemical environment based on thermodynamic and 
56 electrochemical properties. Assuming that there are elements $X(X=A, B, C, \ldots)$ with 57 X-containing compounds, denoted by $\mathrm{X}_{j}\left(1 \leq j \leq N_{X}\right)$, the half-reaction $\mathrm{X}_{j}+(z-j) \mathrm{e}^{-} \rightleftharpoons$ $58 \mathrm{X}_{z}$ holds where $j<z$ and $\mathrm{e}^{-}$denote an electron. A model redox network consists of redox 59 reactions formulated from all possible combinations of two half-reactions that are 60 available in the system (see Supplementary Information, section S.1 and Extended Data

61 Tables 1 and 2). The topological structure of a redox network and the number of 62 possible redox reactions, or links, forming the network $\left(N_{\text {reac }}\right)$ are uniquely determined 63 for a given composition of compounds of elements, or nodes (Fig. 1a, b and Extended 64 Data Table 3). We assumed that there are as many chemotrophic microbial species (a 65 group of species that utilise an identical redox reaction as an energy source, and 66 hereafter referred to as a species (Sp.)) as $N_{\text {reac }}$. The population dynamics of $\mathrm{Sp} . i$, which 67 specifically harness reaction $i$ for population growth is described as:

68

$$
\frac{d M_{i}}{d t}=q_{i}\left(c_{i}\left(-\Delta_{r} G_{i}\right) f_{i}-m_{i}\right) M_{i}
$$

70

71 where $M_{i}$ is the biomass of Sp. $i ;-\Delta_{r} G_{i}\left(\mathrm{~kJ} \mathrm{~mol}^{-1}\right)$ is the negative Gibbs energy change 72 of reaction $\left(-\Delta_{r} G\right)$ of the $i$ th reaction; $f_{i}$ is the microbial catalytic rate of the $i$ th reaction 73 (see Methods); $q_{i}$ is the biomass yield per energy: $c_{i}$ is the fraction of energy that can be 74 used for adenosine triphosphate (ATP) synthesis $\left(0 \leq c_{i} \leq 1\right)$; and $m_{i}$ is the maintenance 75 energy of Sp. $i^{15}$. According to thermodynamics, $-\Delta_{r} G_{1}$ for a reaction with compounds

$76 \quad A_{1}$ and $B_{2}$ as reactants and $A_{2}$ and $B_{1}$ as products in Fig. 1 is given by: 77

$$
-\Delta_{r} G_{1}=-\Delta_{r} G_{1}^{\circ}+R T \ln \frac{\left\{\mathrm{A}_{1}\right\}\left\{\mathrm{B}_{2}\right\}}{\left\{\mathrm{A}_{2}\right\}\left\{\mathrm{B}_{1}\right\}}
$$


80 where $-\Delta_{r} G_{i}$ denotes the negative standard Gibbs energy change of reaction $i$, the value

81 of which is reaction-specific and determined by the compounds associated with reaction

$82 i$ at a given temperature and pressure (see Methods); $R$ is the gas constant; $T$ is the

83 absolute temperature; and $\left\{\mathrm{X}_{j}\right\}$ is the activity of the chemical compound $\mathrm{X}_{j}$. We

84 assumed that the dynamics of the molar concentration of $\mathrm{X}_{j}$ were determined by the

85 input from and output to the surroundings and the abiotic and microbial reactions (see

86 Methods and Supplementary Information, section S.2).

87 Equations 1a and $\mathrm{b}$ have two ecological implications. First, species that

88 harness reactions with higher $-\Delta_{r} G^{\mathrm{o}}$ can obtain larger ATP yields per reactant and,

89 therefore, enjoy larger growth rates. Each redox reaction can be regarded as an

90 ecological niche whose potential quality at the standard condition is given by $-\Delta_{r} G^{\text {o }}$,

91 and a species using a high-quality reaction (high-quality niche) is more likely to grow

92 faster. Second, the population growth rate is modified by the chemical environment, as

93 indicated by the dependence of $-\Delta_{r} G$ on the activity of chemical compounds $\left\{X_{j}\right\}$,

94 with the population growth rate increasing with increasing amounts of reactants or

95 decreasing amounts of products. These formulas provide a basis for understanding

96 chemotrophic community dynamics in terms of community ecological theory.

98 Interspecific interactions in chemotrophic communities and reaction segmentation

99 Interspecific interaction is a key concept in community ecology and provides

100 a mechanistic basis for understanding multi-species population dynamics. The

101 dependence of population growth on the chemical environment, as depicted in equation 
$1021 \mathrm{~b}$, identifies three types of compound-mediated interspecific interactions operating in

103 chemotrophic communities. The first is 'reactant-mediated competition', which is a

104 negative interaction arising between species that consume the same resource (e.g., Sp. 1

105 and 3 in Fig. 1). The second is 'product-mediated competition', which is another kind of

106 negative interaction (and unknown in animal and plant communities) arising between

107 species that produce the same product (e.g., Sp. 2 and 3 in Fig. 1). The third is

108 'mutualism', which is a positive interaction that occurs when a species (the donor)

109 supplies its product to another species (the recipient) as a reactant (e.g., Sp. 1 and 2 in

110 Fig. 1) ${ }^{14,13}$. Note that the positive effect is not only from the donor species to the

111 recipient species; rather, the donor also receives a benefit from the recipient as the

112 removal of product increases $-\Delta_{r} G$ of the donor's energy-source reaction.

113 The model analysis revealed that the three types of interspecific interactions

114 can jointly lead to reaction segmentation, which was identified as a key process for

115 understanding the organisation of chemotrophic communities. A redox reaction can be

116 divided into sequential reactions in the presence of appropriate electron carriers $\left(\mathrm{B}_{1}\right.$ and

$117 \mathrm{~B}_{2}$ in Fig. 1a) or intermediates $\left(\mathrm{A}_{2}\right.$ in Fig. 1b) that mediate electron transfer between the

118 two reactions. When a reaction is segmented, energy production yielded from either

119 subdivided reaction is lower than the overall reaction at the standard condition (see

$120-\Delta_{r} G_{i}{ }^{\mathrm{o}}$ values in Fig. 1). Therefore, it is reasonable to assume that the overall reaction

121 should generate more metabolic energy and, thus, be preferred to either subdivided

122 reaction. However, this is not the case with consortium species (Sp. 1 and 2 in Fig. 1)

123 that use the subdivided reactions and may competitively exclude solitary species (Sp. 3

124 in Fig. 1) that use the overall reaction. The replacement can be explained by the

125 combined effects of the above-mentioned three kinds of interactions. Each consortium 
species is in a competitive relationship (reactant- or product-mediated) with the solitary

127 species, while the two consortium species are in mutualistic relationship, as mentioned

128 above. If the right conditions are met, the consortium species may replace the solitary

129 one, and the overall reaction would be segmented into several subdivided reactions.

130 Reaction segmentation can be regarded as the result of an ecological division of

131 metabolic labour, which enables more efficient resource — and energy — use (see

132 Supplementary Information, section S.4.3 and Extended Data Fig. 3).

\section{Chemical environments and chemotrophic community structure}

135 Ecological theory predicts that resource availability and composition are essential

136 determinants of community structure. The same is true for chemotrophic communities

137 for which redox reactions serve as energy sources, and the structure of the redox

138 network has a substantial impact on consortium formation and the division of metabolic

139 labour. Key parameters that strongly affect the occurrence of the division of metabolic

140 labour are identified through the analysis of model communities with varying chemical

141 environmental conditions.

142 First, the amount of chemical compounds in a system should be large enough

143 for the mutualistic division of metabolic labour to take place. For example, in a model

144 system with $\leq 2$ species at a steady-state, mutualistic consortium is more likely to

145 emerge when compound availability $\left(X_{t o t}\right)$, as measured by the ratio of input rate to

146 output rate, is larger (Fig. 2a; see Methods). This is because compound availability

147 increases the likelihood that sufficient amounts of electron carriers or intermediates,

148 which are necessary to subdivide an energy-providing redox reaction, are present in the

149 system. If compound availability is not large enough, the community will be dominated 
150 by one species or two species that are in a competitive relationship. The same is true for

151 a multi-species system, where the benefit a species receives from the entire community

152 is larger in a community with higher compound availability (Fig. 3a; see Methods for

153 the definition of 'the degree of mutualism').

154 Second, due to the similar reason, the diversity of chemical compounds

155 favours the division of metabolic labour. For model systems with $\geq 2$ species, we set a

156 3-element chemical environment with a varying number of compounds per element (2

157 to 4 compounds each) and evaluated how the community structure responded to the

158 changed compound diversity $\left(N_{t o t}=N_{\mathrm{A}}+N_{\mathrm{B}}+N_{\mathrm{C}}\right)$ (the steady-state number of species

159 at $N_{\text {tot }}$ is summarised in Extended Data Fig. 4). The model analysis revealed that a

160 mutualistic consortium was more likely to be formed in a redox network with higher

161 compound diversity, as suggested by increased mutualism (Fig. 3a). This occurs as a

162 larger $N_{\text {tot }}$ achieves more complex pathways within a metabolic network and increases

163 the likelihood that appropriate electron carriers or intermediates are present in the

164 system.

165 Third, the catalytic effectiveness at which chemotrophs accelerate redox

166 reactions also affects community structure. The maximum catalytic rate constant $\left(r_{i}\right.$; see

167 Methods) is the maximum rate at which a chemotroph catalyses a redox reaction. The

168 model analysis revealed that $r_{i}$ had a considerable effect on the establishment of a

169 mutualistic consortium in a system (Fig. 2b). More specifically, when the maximum

170 catalytic rate constant was higher, the division of metabolic labour was more likely to

171 take place (Fig. 3b). This can be explained by the coupling strength between

172 chemotrophic community dynamics and chemical reactions. For a consortium species to

173 outcompete a solitary species, the mutualism-driven positive feedback between 
consortium species should be strong enough. The maximum catalytic rate constant

175 determines how strongly a consortium species can enhance the population growth of the

176 other consortium species and, therefore, can strongly affect the competitive outcome

177 and the emergence of the division of metabolic labour.

178

179 Ecosystem productivity enhanced by mutualistic consortiums

180 Segmentation of the reaction pathway caused by the mutualistic division of

181 metabolic labour can improve energy acquisition efficiency at the community level and

182 enhance ecosystem productivity. In both systems with $\leq 2$ species and multi-species, the

$183-\Delta_{r} G_{i}^{\mathrm{o}}$ values of the reactions used by chemotrophic species were smaller when the

184 mutualistic division of labour was favoured (larger $X_{\text {tot }}$ or larger $N_{\text {tot }}$ ) (Fig. 3c and

185 Extended Data Fig. 5). This is because the establishment of mutualistic relationships

186 enables the chemotrophic community to persist with reactions that have a smaller

187 energy production in the standard state $\left(-\Delta_{r} G_{i}{ }^{0}\right)$. That is, reactions with smaller

$188-\Delta_{r} G_{i}{ }^{\mathrm{o}}$, which are left unused when non-mutualistic, solitary species dominate $\left(X_{t o t}=\right.$

$18910^{-4}$ in Fig. 3c), can be efficiently utilised when a mutualistic consortium is established.

190 As a result, ecosystem productivity, as measured by the median of the total biomass, is

191 also improved under conditions that favour the mutualistic division of metabolic labour

192 (Fig. 3d).

193

194 Discussion

195 We have shown how the chemotrophic community is organised under the

196 thermodynamic rules that govern chemical reactions and energy transformations. The

197 eco-geochemical interplay gives rise to three kinds of interspecific interactions: 
reactant-mediated and product-mediated competitions and mutualism. These lead to the

199 key process of the division of metabolic labour, which subdivides an energy-providing

200 redox reaction into several segmented reactions and, as a result, increases complexity in

201 a reaction network and is accompanied by increased ecosystem productivity and

202 microbial diversity. It is the explicit incorporation of free energy in formulating the

203 chemotrophic dynamics that reveals the ecological properties of the chemotrophic

204 community.

205 Giving consideration to eco-geochemical interplay is essential to

206 understanding why some redox reactions are favoured, while other are not. Redox

207 reactions are regarded as niches for chemotrophic species. Therefore, one might expect

208 that a species would favour a reaction with a high $-\Delta_{r} G^{\mathrm{o}}$ as its energy source. However,

209 the redox reaction network is shaped through niche construction processes such that

210 utilization of one reaction changes the amount of energy available from other reactions,

211 i.e., not $-\Delta_{r} G^{\mathrm{o}}$ but $-\Delta_{r} G$. Therefore, as shown by the model analysis, reactions with

212 relatively low $-\Delta_{r} G^{\mathrm{o}}$ could be more favoured due to the biological niche construction

213 effect. The interplay between chemotrophs and the chemical environment might explain

214 why cultivation techniques have not been established for some chemotrophs, especially

215 chemolithoautotrophs living in oligotrophic environments. What may be necessary for

216 successful cultivation might not only be unknown physicochemical conditions, but also

217 the co-presence of key species that support the survival of the target species through the

218 modification of the redox network.

219 The division of metabolic labour can be regarded as a kind of niche

220 partitioning. Yet, it is fundamentally different from those in animal or plant

221 communities in that, due to the nature of the redox network, it is accompanied by the 
establishment of mutualistic interactions. The mutualistic nature of the division of

223 metabolic labour has implications for the classic issue concerning the relationship

224 between community complexity and ecosystem functioning. The model community of

225 chemotrophic microbes demonstrates that chemotrophs can acquire energy more

226 efficiently, and the total biomass becomes higher, when the number of compounds or

227 species is larger. This suggests that the interplay between chemotrophs and chemical

228 energy can act as a mechanism for a positive complexity-productivity relationship to

229 emerge. A complex metabolic network with more subdivided pathways may increase

230 the stability of chemotrophic ecosystems because the fragmentation of a metabolic

231 network is inseparable from increases in mutualistic interactions and the subsequent

232 enhanced productivity.

233 The present study explored the basic nature underlying how a chemotrophic

234 community in an anaerobic environment is organised via the explicit consideration of

235 thermodynamic constraints. Further model verification and extension will help us to

236 understand more specific inter-relationships between microbial and biogeochemical

237 networks. Aerobic microbes that gain energy from aerobic respiration with a high

$238-\Delta_{r} G_{i}^{\mathrm{o}}$ appear to be dominant under the high availability of organic matter and oxygen,

239 which may be further addressed by investigating the effect of the availability of specific

240 compounds on a microbial community. Moreover, the redox reactions available for ATP

241 production are not only limited by thermodynamics but by kinetics (e.g., activation

242 energy) and other physico-chemical properties of compounds (e.g., oxygen toxicity)

243 related to the reaction ${ }^{16}$.

\section{References}


246 1. Parkes, R. J. et al. Deep bacterial biosphere in Pacific Ocean sediments. Nature $247 \quad 371,410-413(1994)$.

248 2. Inagaki, F. et al. Exploring deep microbial life in coal-bearing sediment down to $249 \sim 2.5 \mathrm{~km}$ below the ocean floor. Science 349, 420-424 (2015).

250 3. Magnabosco, C. et al. The biomass and biodiversity of the continental subsurface. Nature Geoscience 11, 707-717 (2018).

4. Jørgensen:2012. Shrinking majority of the deep biosphere. Proc. Natl. Acad. Soc. 109, 15976-15977 (2012).

254 5. Bar-On, Y. M., Phillips, R. \& Milo, R. The biomass distribution on Earth. Proceedings of the National Academy of Sciences 115, 6506-6511 (2018).

6. Kallmeyer, J., Pockalny, R., Adhikari, R. R., Smith, D. C. \& D’Hondt, S. Global distribution of microbial abundance and biomass in subseafloor sediment. Proceedings of the National Academy of Sciences of the United States of America 109, 16213-16216 (2012).

2607 Flemming, H. C. \& Wuertz, S. Bacteria and archaea on Earth and their abundance in biofilms. Nature Reviews Microbiology 17, 247-260 (2019).

262 8. Jørgensen, B. B. \& Boetius, A. Feast and famine - Microbial life in the deep-sea 263 bed. Nature Reviews Microbiology 5, 770-781 (2007).

$2649 . \quad$ Colman, D. R., Poudel, S., Stamps, B. W., Boyd, E. S. \& Spear, J. R. The deep, 265 hot biosphere: Twenty-five years of retrospection. Proceedings of the National Academy of Sciences of the United States of America 114, 6895-6903 (2017).

267 10. Stevens, T. Lithoautotrophy in the subsurface. FEMS Microbiology Reviews 20, 268 $327-337$ (1997).

269 11. Momper, L., Jungbluth, S. P., Lee, M. D. \& Amend, J. P. Energy and carbon 
metabolisms in a deep terrestrial subsurface fluid microbial community. ISME Journal 11, 2319-2333 (2017).

272 12. Bradley, J. A. et al. Widespread energy limitation to life in global subseafloor 273 sediments. Science Advances 6, 1-9 (2020).

274 13. Seto, M. \& Iwasa, Y. The fitness of chemotrophs increases when their catabolic by-products are consumed by other species. Ecology Letters 22, 1994-2005 (2019).

14. Seto, M. \& Iwasa, Y. Microbial material cycling, energetic constraints and ecosystem expansion in subsurface ecosystems. Proceedings of the Royal Society B: Biological Sciences 287, 20200610 (2020).

15. Seto, M. \& Iwasa, Y. Population dynamics of chemotrophs in anaerobic conditions where the metabolic energy acquisition per redox reaction is limited. Journal of Theoretical Biology 467, 164-173 (2019).

16. Bar-Even, A., Flamholz, A., Noor, E. \& Milo, R. Rethinking glycolysis: On the biochemical logic of metabolic pathways. Nature Chemical Biology 8, 509-517 (2012).

286

17. Bae, W. \& Rittmann, B. E. A structured model of dual-limitation kinetics. Biotechnology and Bioengineering 49, 683-689 (1996).

\section{Figure Legends}

290 Fig. 1 | Structures of redox networks and examples of metabolic interactions. a,

291 When $\left(N_{A}, N_{B}, N_{C}\right)=(2,2,2)$ and $\mathbf{b}$, when $\left(N_{A}, N_{B}, N_{C}\right)=(3,2,0)$. The number $i$ in the 292 circle stands for the $i$ th reaction and species. Blue and red arrows correspond to 293 oxidation and reduction reactions, respectively. The right panel illustrates the half- 
reactions that build overall redox reactions 1, 2, and 3 (see Supplementary Information, section S.1) and the metabolic relationships among species 1, 2, and 3.

297 Fig. 2 The number and relationships of species when $\left(N_{A}, N_{B}, N_{C}=\mathbf{2 ,}, \mathbf{2}, \mathbf{2}\right)$, a, The 298 steady-state responses to the number of compounds $X_{\text {tot }}$, and $\mathbf{b}$, the maximum catalytic 299 rate constant, $r_{i}$. During the simulation in (b), $r_{i}$ for all species was fixed to the same 300 value. 0 and 1 meant that either no species or 1 species existed in a steady-state, 301 respectively. Two species existed in a steady state for cases $2 \mathrm{C}$ and $2 \mathrm{M}$ and could either 302 compete for the same resource and produce the same compound (C) or mutually divide 303 the metabolic labour (M).

Fig. 3 | Steady state responses to changes in the availability of compound $X_{t o t}$, and the number of compounds, $N_{\text {tot }}$ a, The degree of mutualism (see Methods for the definition of 'the degree of mutualism'), $\mathbf{b}$ and $\mathbf{c}$ are the median of $-\Delta_{r} G_{i}$ of the energysource reactions and the maximum catalytic rate, $r_{i}$, of all species surviving at a steadystate with other species. $\mathbf{d}$, the median of the total biomass, $\sum M_{i}^{*}$, for 1000 iterations.

\section{Methods}

312 An algorithm for formulating redox reactions and constructing a redox network 313 model

We developed an algorithm in the Wolfram language in Mathematica 12, which automatically formulates redox reactions when all of the numbers of the compounds of $\mathrm{X}$ 316 element, $N_{\mathrm{X}}$, are provided (see Supplementary Information, section S.3). The programs 317 are available in the Programs folder. By running the processes 1 to 3 in Simulation.nb: i) 
318 the standard Gibbs energy change of the compounds is randomly selected, ii) half 319 reactions with corresponding standard reduction potentials are formulated (Extended 320 Data Table 1), and iii) redox reactions are generated for all of the possible combinations 321 of two half reactions (Extended Data Table 2). The number of possible redox reactions, $322 N_{\text {reac }}$, were uniquely determined depending on the number of compounds of elements 323 (Extended Data Table 3).

The microbial catalytic rate and the standard Gibbs energy change of formation was determined by the consumption rate of compound $\mathrm{X}_{j}$ in the first term of the left side of its energy-source redox reaction. When a species harnesses a disproportionation reaction, the microbial catalytic rate follows the Monod (or Michaelis-Menten) equation. Using reaction 9 in Extended Data Table 2 as an example,

$$
f_{9}=r_{9} \frac{\left[\mathrm{A}_{2}\right]}{K_{9 \mathrm{~A}_{2}}+\left[\mathrm{A}_{2}\right]}
$$

where $\left[\mathrm{X}_{j}\right]$ denotes the molar concentration of $\mathrm{X}_{j}, r_{9}$ is the maximum catalytic rate constant of Sp. $9\left(\mathrm{~mol} \mathrm{mg}^{-1} \mathrm{~h}^{-1}\right)$, and $K_{9 X_{j}}$ is the half-saturation constant for $\mathrm{X}_{j}$ of Sp. 9. For species harnessing the rest of the redox reactions, their microbial catalytic rates follow the dual Monod equation ${ }^{17}$. Using reaction 1 in Extended Data Table 2 as an example,

$$
f_{1}=r_{1} \frac{\left[\mathrm{A}_{1}\right]}{K_{1 \mathrm{~A}_{1}}+\left[\mathrm{A}_{1}\right]} \frac{\left[\mathrm{A}_{3}\right]}{K_{1 \mathrm{~A}_{3}}+\left[\mathrm{A}_{3}\right]}
$$


341 The standard Gibbs energy change of the $i$ th reaction, $-\Delta_{r} G_{i}^{\text {o }}$, is equal to the sum of

342 the standard Gibbs energy change of formation of reactants minus that of the products.

343 Thus, for reaction 1 in Extended Data Table 2,

$$
-\Delta_{r} G_{i}^{\circ}=\Delta_{f}{G_{\mathrm{A}_{1}}}^{\circ}+\Delta_{f}{G_{\mathrm{A}_{3}}}^{\circ}-2 \Delta_{f} G_{\mathrm{A}_{2}}^{\circ}
$$

where $\Delta_{f} G_{X j}{ }^{\mathrm{o}}$ denotes the standard Gibbs energy change of formation of compound $\mathrm{X}_{j}$.

The dynamics of the molar concentration of a compound $X_{j}$, follows

[Microbial supply rate] - [Microbial consumption rate $]-D_{X_{j}} X_{j}$

where $I_{X j}$ and $D_{X j}$ are the inflow rate $\left(\mathrm{mol} \mathrm{L}^{-1} \mathrm{~h}^{-1}\right)$ and the outflow rate constant $\left(\mathrm{h}^{-1}\right)$ of $\mathrm{X}_{j}$, respectively. $X_{j}$ is abiotically and microbially supplied and consumed, and the abiotic reaction rate is proportional to the product of the molar concentration of the reactants. The microbial catalytic rate is given by the product of $f_{i} M_{i}$ and the stoichiometry constant.

Default values of the parameters and the initial conditions of the variables

362 Data Table 4 summarises the symbols, definitions, units, values of the initial conditions

363 of the variables, and the default values of the parameters. The values of the standard 
364 Gibbs energy change of formation of compound $\mathrm{X}_{j}, \Delta_{f} G_{X j}{ }^{\mathrm{o}}$, the maximum reaction rate 365 constant of the $i$ th species, $r_{i}$, and the abiotic reaction rate constant of forward reactions $366 k_{i}$, were regarded as random variables (see Supplementary Information, sections S.1 and

367 S2 for the abiotic and backward reactions). The values of $\Delta_{f} G_{X j}{ }^{\mathrm{o}}, r_{i}$, and $k_{i}$ in $\log _{10}$ were 368 assumed to follow a uniform distribution within the range shown in Extended Data 369 Table 4. They were generated by the RandomReal function and were eventually 370 converted to antilog values. An example of the dynamics of the model when $\left(N_{\mathrm{A}}, N_{\mathrm{B}}\right.$, $\left.371 N_{\mathrm{C}}\right)=(3,3,3)$ is shown in Extended Data Fig. 1a (see Supplementary Information, 372 section S.4.1). Within the given range of values of the parameters, the inflow and 373 outflow rates of compound $\mathrm{X}_{j}$ significantly exceeded the abiotic reaction rates in the system. Consequently, the molar concentration of compounds in the system without microbes $\left(M_{i}=0\left(1 \leq i \leq N_{\text {reac }}\right)\right)$ was almost determined by the balance between inflow and outflow. The molar concentration of compounds at the abiotic steady state was $X_{j}=$ $I_{X_{j}} / D_{X_{j}}$. We defined the availability of compounds as $X_{t o t}=\sum I_{X_{j}} / D_{X_{j}}$. The simulations at the given compound diversity $N_{t o t}=N_{\mathrm{A}}+N_{\mathrm{B}}+N_{\mathrm{C}}$ and $X_{\text {tot }}$ condition were repeated 1000 times and only those results that reached a steady-state were extracted. $N_{t o t}=6,7,8,9,10,11$, and 12 in Fig. 3 correspond to $\left(N_{A}, N_{B}, N_{C}\right)=(2$, $2,2),(3,2,2),(3,3,2),(3,3,3),(4,3,3),(4,4,3)$, and $(4,4,4)$, respectively. Although the system had multi-stability since the microbial community at the steady-state depends on the invasion history (see Supplementary Information, section S.4.2), the 384 same initial conditions were used to obtain the steady states.

\section{The degree of mutualism}

387 To assess the degree of mutualism of a community at a given $N_{t o t}$ and $X_{\text {tot }}$ condition, and 
for all of the species that could exist at a steady state for iterations, we evaluated the

389 steady-state biomass of Sp. $i$ when it was solely present in a redox network, $\overline{M_{l}}$, and the

390 steady-state biomass of Sp. $i$ when it exists with other species in the redox network with

391 the same structure and abiotic settings, $M_{i}^{*}$. If $\frac{\overline{M_{l}}}{M_{i}^{*}}<1$, the steady-state biomass of Sp. $i$

392 was collectively, positively affected and supported by the presence of other species. The

393 degree of mutualism of a community was defined as the ratio of Sp. $i$ that satisfied $\frac{\overline{M_{l}}}{M_{i}^{*}}$

$394<1$ for all species surviving at a steady state with other species for iterations.

395

\section{Data availability}

The parameters and differential equations for the NDsolve function for numerical differentiation in Mathematica that generated Extended Data Figure 1a are

399 provided in the Programs/FigS1/data folder. The default values of the parameters and initial conditions of the variables are summarised in Extended Data Table 4.

401

402

\section{Code availability}

All codes were written in the Wolfram Language platform using Mathematica

404 12. The codes are available in the Programs folder. See details in Supplemental

405 Information S.3.

406

407 Acknowledgements

408

MS is supported by JSPS Grant-in-Aid for Scientific Research (C) Grant

409 Number 19K06853. MK is supported by JSPS KAKENHI (JP19H05641, JP21H05315)

410 and the Environment Research and Technology Development Fund 
411 (JPMEERF20214103) of the Environmental Restoration and Conservation Agency of

412 Japan. We thank the following people for their comments: Y. Iwasa, K. Kadowaki, and 413 Y. Tachiki.

Author contributions

M.S and M.K conceived of the presented idea. M.S designed the model and

417 the computational framework and analysed the data. M.S and M.K contributed equally

418 to the interpretation of the results and writing the manuscript.

Competing interests

The authors declare no competing interests.

Supplementary Information is available for this paper.

Correspondence and requests for materials should be addressed to Mayumi Seto

\section{Peer review information}

428

Reprints and permissions information is available at www.nature.com/reprints

429

430 Extended data figures and tables

431 Extended Data Figure 1 | An example of the dynamics of microbes and the Gibbs

432 energy when $\left(\boldsymbol{N}_{\boldsymbol{A}}, \boldsymbol{N}_{\boldsymbol{B}}, \boldsymbol{N}_{\boldsymbol{C}}\right)=(\mathbf{3}, \mathbf{3}, \mathbf{3})$. a, The dynamics of the biomass of species $i(1 \leq$ $433 i \leq 60), M_{i} . \mathbf{b}$, The dynamics of the biomass of five species (top) and the negative Gibbs 434 energy change of reaction (bottom) when species survive at a steady state when (a) 
436 the parameters were set as the default values listed in Extended Data Table 4. The

437 values of random parameters are listed in Program/FigS1/data.

Extended Data Figure 2 | Simulation when a species is randomly and sequentially

added to a system. a, The flowcharts represent Steps 2 and 3 in Supplementary

Information, section S.4.2. b, The identifier number of species surviving at $t\left(x_{i}(t)>\right.$ $10^{-6}$ ) when each species was randomly selected and added to the system in order every 1000 hours when $\left(N_{A}, N_{B}, N_{C}\right)=(4,4,4)$ and $X_{\text {tot }}=1$. Filled circles denote species when no more species could replace them. Black, blue, and red show the results of the trials out of 10. b (left), The same combination of species exists at a steady state, suggesting that there is a single, stable, steady state. $\mathbf{b}$ (right), The combination of species existing in a steady state depends on the invasion history, suggesting the presence of multi-

448 stable, steady-states.

Extended Data Figure 3 | The combinations of species surviving in a steady state (a, b, and c) and $R_{s}^{*} / R_{c}{ }^{*}(\mathrm{~d})$ in response to various $\left(I_{\mathrm{B} 1}, I_{\mathrm{B} 2}\right)$ conditions when $\left(N_{A}\right.$, $\left.\left.\boldsymbol{N}_{\boldsymbol{B}}, \boldsymbol{N}_{\boldsymbol{C}}\right)=(\mathbf{2}, \mathbf{2}, \mathbf{2})\right)$. The energy-source reactions of species (Sp.) 1, 2, and 3 are reactions 1, 2, and 3 in Fig. 1(a), respectively. a, Sp. 3 is solely present at $t=0$. b, Sp. 1 and 2 are present at $t=0$. c, Sp. 1, 2, and 3 are present at $t=0$. In (a-c), black: Sp. 3 only exists; pink: Sp. 1 only exists; cyan: Sp. 2 only exists; blue: Sp. 1 and 2 exist; green: Sp. 1 and 3 exist; red: Sp. 2 and 3 exist. Sp. 1 and 2 were unable to exist in the white region in (b) and (d). We let $R^{*}=A_{1}{ }^{*} C_{2}{ }^{*}$ where * denotes the steady state. $R^{*}$ 458 when Sp. 3 only exists is referred to as $R_{s}{ }^{*}$ and when Sp. 1 and 2 exist it is referred to as 
$R_{c}{ }^{*}$, respectively. $s$ stands for solitary (Sp. 3) and $c$ stands for the two consortium

460 species (species 1 and 2). The solid contour in (d) shows $R_{s}{ }^{*} / R_{c}{ }^{*}=1$, above which

461 species 2 and 3 can lower the steady state abundance of $A_{1}{ }^{*} C_{2}{ }^{*}$ more than species 3 can 462 alone.

463

Extended Data Figure 4 | The steady-state number of species at different total number of compounds, $\boldsymbol{N}_{\text {tot }}$ and compound availability, $\boldsymbol{X}_{t o t} \cdot N_{t o t}=6,7,8,9,10,11$, and 12 correspond to $\left(N_{A}, N_{B}, N_{C}\right)=(2,2,2),(3,2,2),(3,3,2),(3,3,3),(4,3,3),(4,4$, $3)$, and $(4,4,4)$, respectively. Filled circles show the median and the solid and dotted error bars show the 25 and 75 percentiles and the minimum and maximum values.

Extended Data Figure $5 \mid$ The distribution of $-\Delta_{r} G_{i}{ }^{0}$ from the energy-source reactions of species $i$ surviving in a steady state when $\left(N_{A}, N_{B}, N_{C}=2,2,2\right)$. Decreases in $-\Delta_{r} G_{i}^{\mathrm{o}}$ as $X_{\text {tot }}$ increases means species existing in a steady-state harness reactions with lower $-\Delta_{r} G_{i}$ due to the division of metabolic labour.

Extended Data Table 1 | Half reactions and overall cell reactions with a half reaction as a reduction reaction and the standard hydrogen electrode as an oxidation reaction. $N_{A}=3, N_{B}=2$, and $N_{C}=2$

Extended Data Table 2 | Possible redox reactions when $\left(N_{A}, N_{B}, N_{c}\right)=(3,2,2)$. The standard Gibbs energy change of reactions were calculated by setting $\Delta_{f} G_{\mathrm{A} 1}{ }^{\mathrm{o}}=9.71$, $\Delta_{f} G_{\mathrm{A} 2}{ }^{\mathrm{o}}=135.47, \Delta_{f} G_{\mathrm{A} 3}{ }^{\mathrm{o}}=409.13, \Delta_{f} G_{\mathrm{B} 1}{ }^{\mathrm{o}}=53.1, \Delta_{f} G_{\mathrm{B} 2}{ }^{\mathrm{o}}=155.25, \Delta_{f} G_{\mathrm{C} 1}{ }^{\mathrm{o}}=70.33$, and 
484 Extended Data Table 3 | The total number of possible redox reactions at a given $\left(N_{A}\right.$, $\left.485 N_{B}, N_{c}\right)$ condition

486

487 Extended Data Table 4 | Symbols, definition, units, values of the initial conditions, 488 and default values or ranges of the parameters 
Figure 1

(a)<smiles>CCOCCC(C)OCC</smiles>

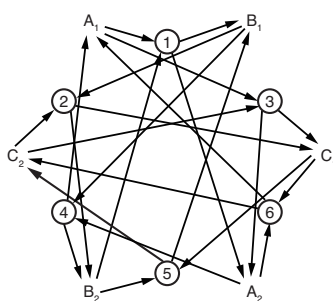

(b)
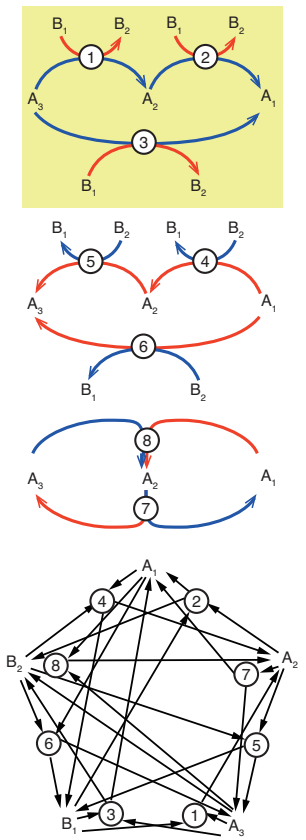

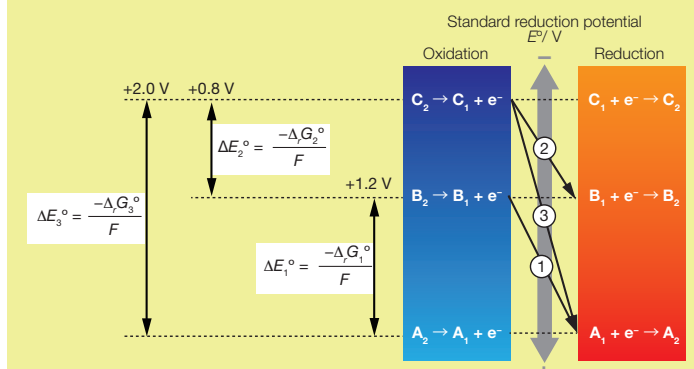

$\begin{array}{lcc}\text { Energy-source } & -\Delta G^{\circ} & -\Delta G \\ \text { redox reaction } & \left(\mathrm{kJ} \mathrm{mol}^{-1}\right) & \left(\mathrm{KJ} \mathrm{mol}^{-1}\right)\end{array}$
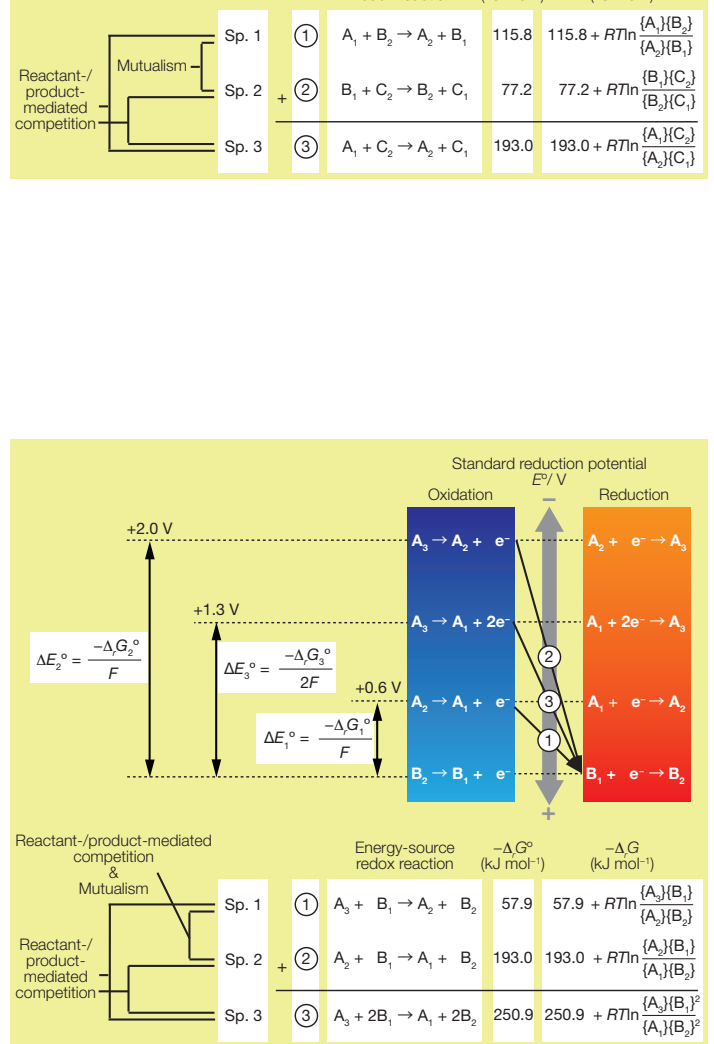

Figure 1: Structures of redox networks and examples of metabolic interactions. a, When $\left(N_{A}, N_{B}, N_{C}\right)=(2,2,2)$ and $\mathbf{b}$, when $\left(N_{A}, N_{B}, N_{C}\right)=(3,2,0)$. The number $i$ in the circle stands for the $i$ th reaction and species. Blue and red arrows correspond to oxidation and reduction reactions, respectively. The right panel illustrates the half-reactions that build overall redox reactions 1,2 , and 3 (see Supplementary Information, section S.1) and the metabolic relationships among species 1,2 , and 3 . 
Figure 2

(a)

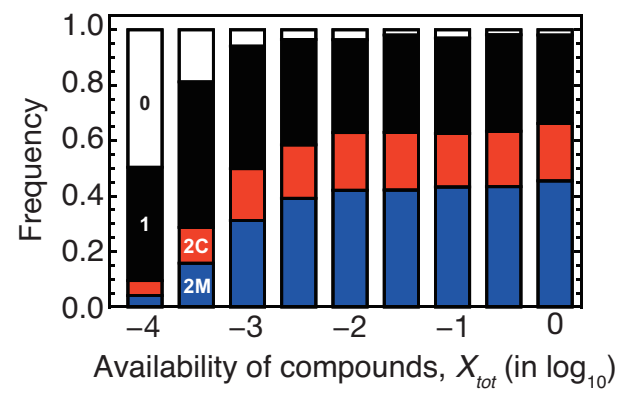

(b)

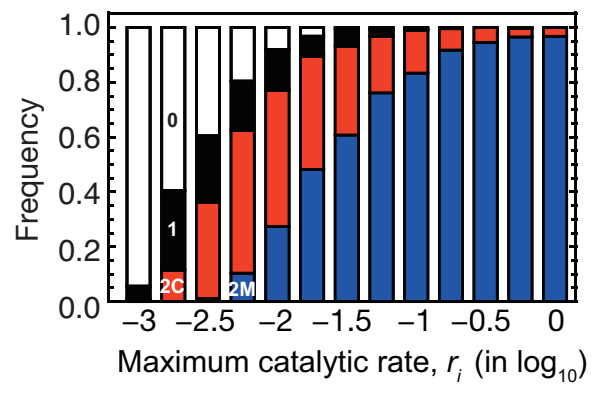

Figure 2: The number and relationships of species when $\left(N_{A}, N_{B}, N_{C}=2,2,2\right)$. a, The steady-state responses to the number of compounds $X_{t o t}$, and $\mathbf{b}$, the maximum catalytic rate constant, $r_{i}$. During the simulation in (b), $r_{i}$ for all species was fixed to the same value. 0 and 1 meant that either no species or 1 species existed in a steady-state, respectively. Two species existed in a steady state for cases $2 \mathrm{C}$ and $2 \mathrm{M}$ and could either compete for the same resource and produce the same compound $(\mathrm{C})$ or mutually divide the metabolic labour $(\mathrm{M})$. 
Figure 3

(a) Degree of mutualism of a community $\begin{array}{llll}0.2 & 0.4 & 0.6 & 0.8\end{array}$
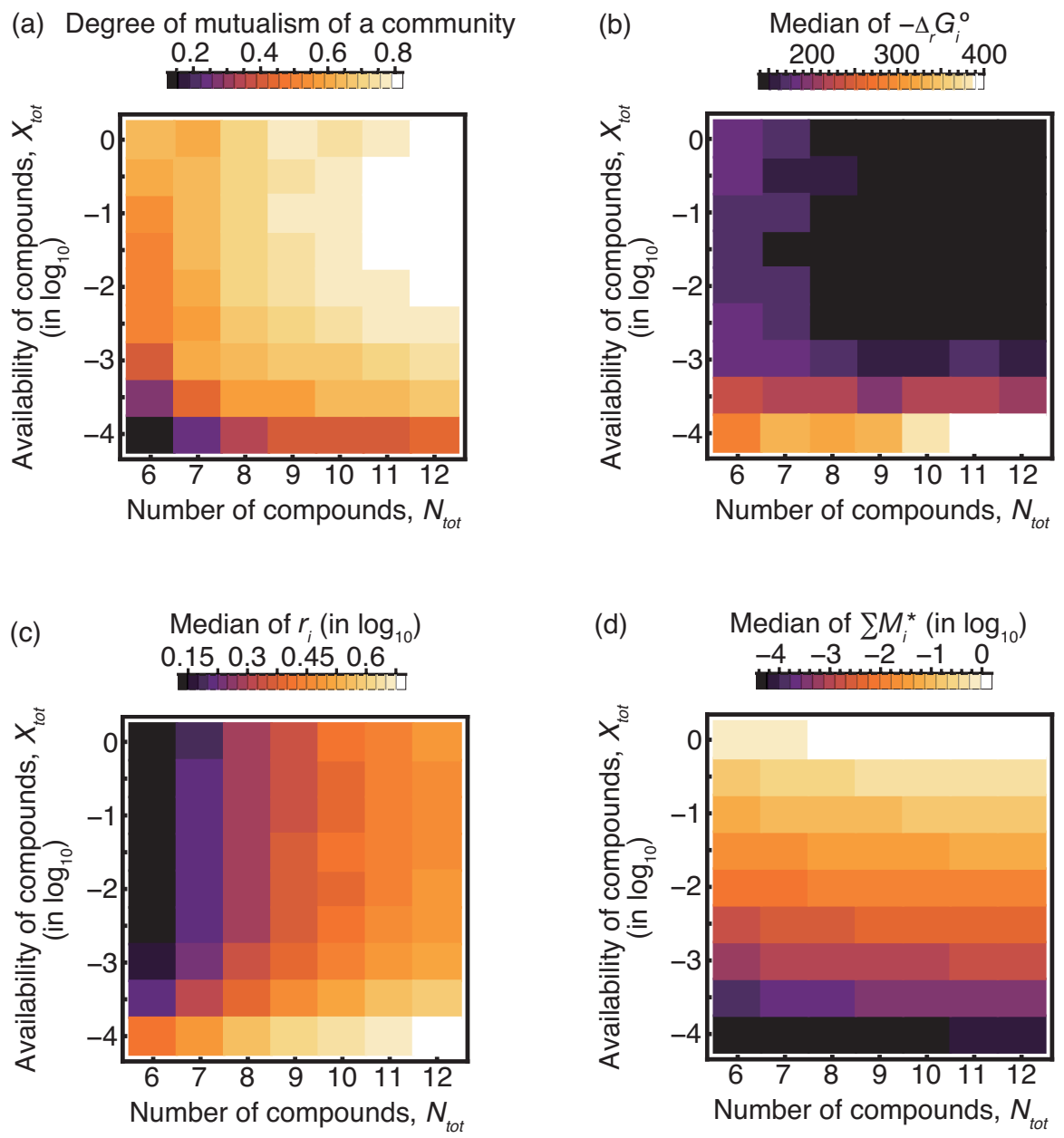

Figure 3: Steady state responses to changes in the availability of compound $X_{t o t}$, and the number of compounds, $N_{t o t}$. a, The degree of mutualism (see Methods for the definition of 'the degree of mutualism' ), $\mathbf{b}$ and $\mathbf{c}$ are the median of $-\Delta_{r} G_{i}^{\circ}$ of the energy-source reactions and the maximum catalytic rate, $r_{i}$, of all species surviving at a steady-state with other species. d, the median of the total biomass, $\sum M_{i}^{*}$, for 1000 iterations. 


\section{Supplementary Files}

This is a list of supplementary files associated with this preprint. Click to download.

- ExtendedDataTables.docx

- SIGuide.docx

- Supplementallnfo.docx

- ExtendedDataFigures.pdf 